\title{
Revisiting Angle Surgery for Management of Open-angle Glaucoma: Ab Interno Trabeculotomy
}

\author{
Don Minckler \\ University of California Irvine, Irvine, CA, USA, e-mail: minckler@uci.edu
}

\section{INTRODUCTION}

New ab interno and ab externo approaches to angle surgery in adults and children with open-angle glaucoma show promise for improving intraocular pressure (IOP) control less invasively and with fewer complications than current ab externo filtering procedures. These include the Trabectome, ${ }^{\mathrm{TM}}$ Science canaloplasty, Glaukos, ${ }^{\mathrm{TM}}$ Laser goniopuncture and Solx.

\section{AB INTERNO TRABECULOTOMY}

The Trabectome ${ }^{\mathrm{TM}}$ permits ab interno trabeculotomy which involves ablation of a strip of trabecular meshwork and inner wall of Schlemm's canal with simultaneous aspiration and irrigation to remove tissue debris with a stable anterior chamber. ${ }^{1,2}$ The device includes a ceramic coated insulated footplate that acts as a glide within Schlemm's and also functions to protect adjacent tissues from heat or mechanical injury. ${ }^{3}$

\section{Technique}

This procedure is done via a $1.6 \mathrm{~mm}$ clear corneal incision temporally under direct gonioscopic control utilizing a modified Swan-Jacob goniolens with the goal of opening a 90 to $120^{\circ}$ arc of Schlemm's canal so that aqueous has direct access to collector channels.

The procedure is performed using Trabectome ${ }^{\mathrm{TM}}$ (Fig. 1) approved by the US FDA. The trabectome handpiece (Fig. 2) is a disposable device that incorporates bipolar microelectrocautery for safe ablation and removal of trabecular meshwork, unroofing Schlemm's canal and exposing the natural drainage pathway of the eye (collector channels) to aqueous humor with simultaneous irrigation and aspiration. It has got a footplate at the tip which protects collector channels. A foot pedal control activates aspiration and ablation.

The most useful anatomic landmarks for identifying Schlemm's intraoperatively include the scleral spur and pigmented meshwork, if present. Alternatively, blood in Schlemm's canal after installation of viscoelastic into the anterior chamber often clearly marks the location of Schlemm's. The inferior nasal quadrant is specifically targeted as collector channels are thought most numerous there. As ablation proceeds, the back wall of Schlemm's appears as a white band in the trail of the instrument's footplate. Back bleeding from exposed collector channels or Schlemm's canal typically occurs during the later part of canal opening or when IOP drops as the instrument is removed. Back bleeding typically stops spontaneously over minutes or when an internal tamponade via fluid or an air bubble is installed. Figures 3 and 4 show histopathology of tissue structure from in vitro corneal rim after ab interno trabeculotomy.

Only rarely among the nearly 400 procedures so far performed and reported by the authors, has the resulting hyphema been more than 10 to 20 percent or persisted for more than a few days or been associated with a postoperative IOP spike. Intraoperative back bleeding is probably decreased by preoperative use of apraclonidine. A single 10-0 nylon or polyglactin suture is placed across the corneal wound. Postoperatively, most eyes are treated with 1 to 2 percent pilocarpine for two weeks and preoperative medications resumed temporarily pending IOP results over ensuing weeks.

Reduction of IOP in an ongoing prospective case series involving nearly 400 eyes has averaged 40 percent (mean preoperative IOP of $24 \mathrm{~mm} \mathrm{Hg}$ ) to mid-teen levels (mean postoperative IOP of $16 \mathrm{~mm} \mathrm{Hg}$ ) persisting for at least 40 months in 15 patients. Overall topical medications have been reduced from a preoperative mean of 2.7 to a postoperative mean of 0.8 .

\section{Advantages and Disadvantages of Trabectomy}

To date disadvantages of the Trabectome ${ }^{\mathrm{TM}}$ include only single use of the handpiece and IOP outcomes in the mid-teens, limiting its use to patients with mid-teen IOP goal ranges.

Its advantages include short surgical times, simplified postoperative follow-up, no bleb formation or late infection risk, and no damage to conjunctiva precluding standard surgery thereafter, if necessary. Thus far progression of cataract in phakic eyes has been minimal, also in sharp contrast to standard filtering procedures. Complications in general, other than expected back-bleeding, have been minimal and nonvisionthreatening. 

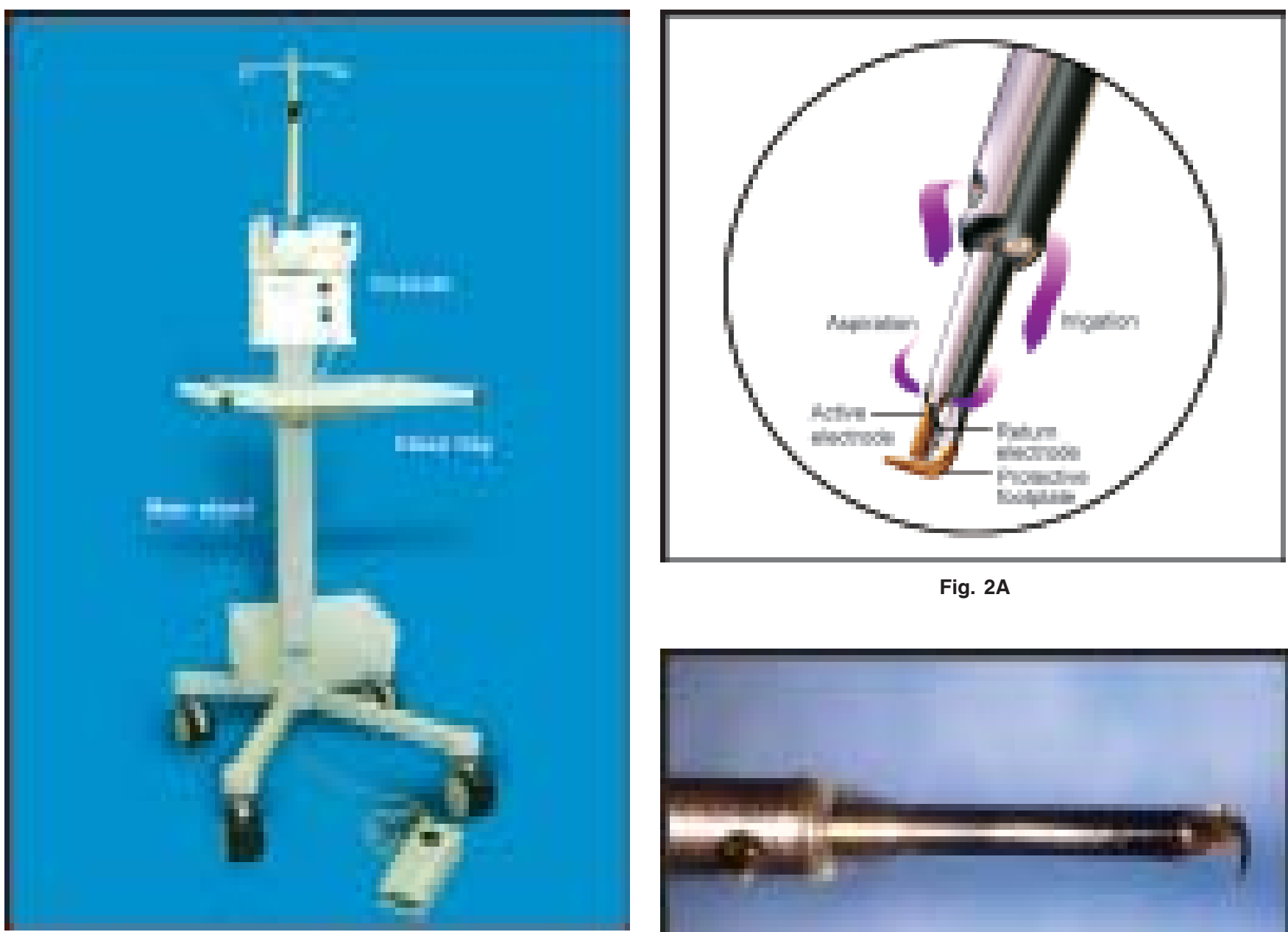

Fig. 2A

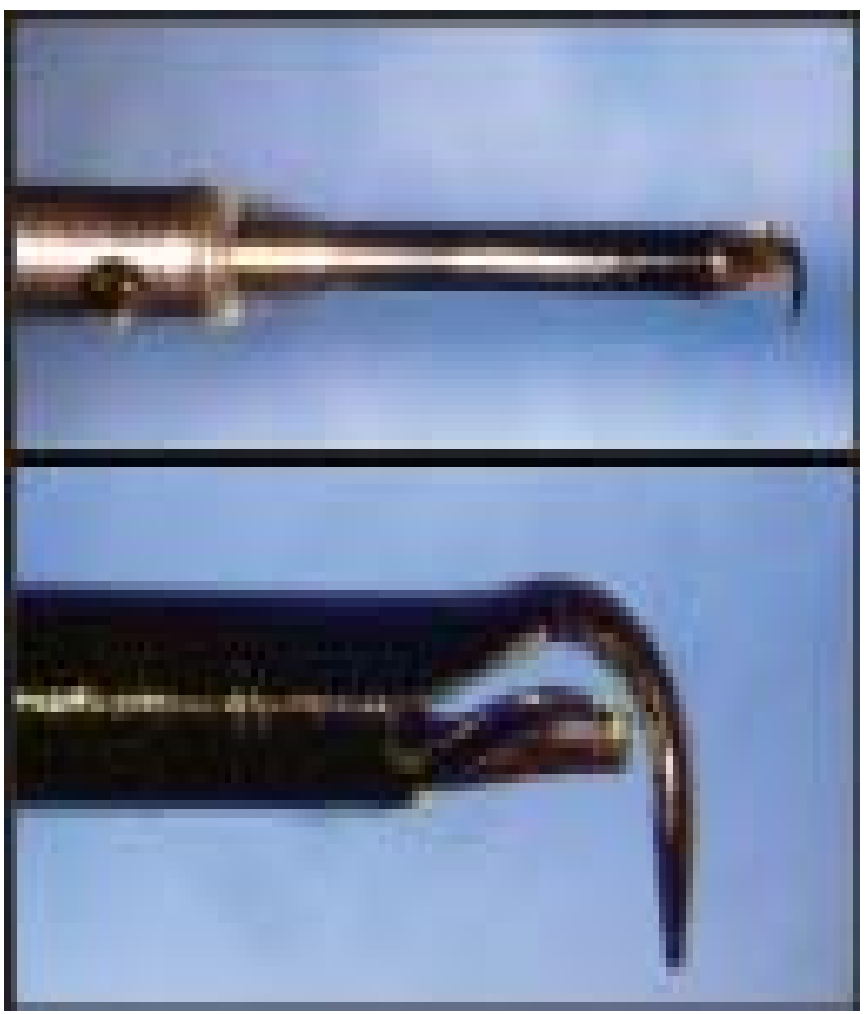

Figs 2A and B: Trabectome handpiece

In theory, this procedure should improve outcomes in children compared to ab externo trabeculotomy or goniotomy but the clinical experience to date has been too minimal for

Figs 1A and B: Trabectome ${ }^{\mathrm{TM}}$ comparative assessment. 

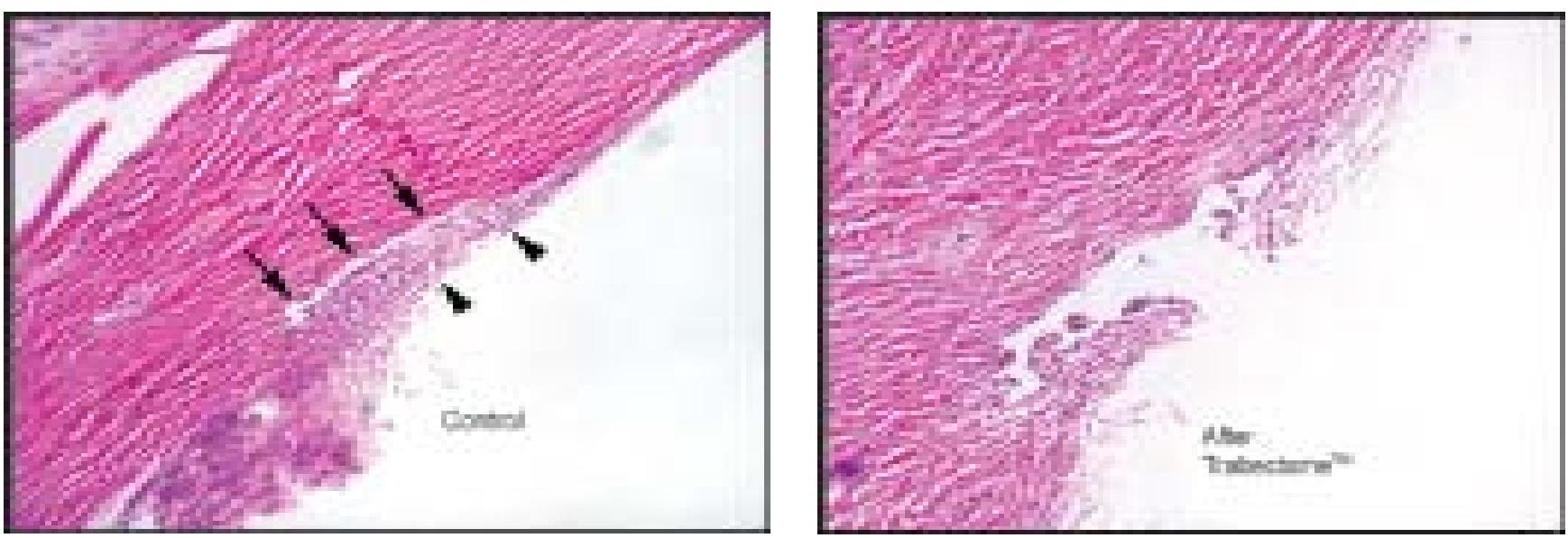

Figs 3A and B: Histopathology showing tissue structure from in vitro corneal rim after ab interno trabeculotomy

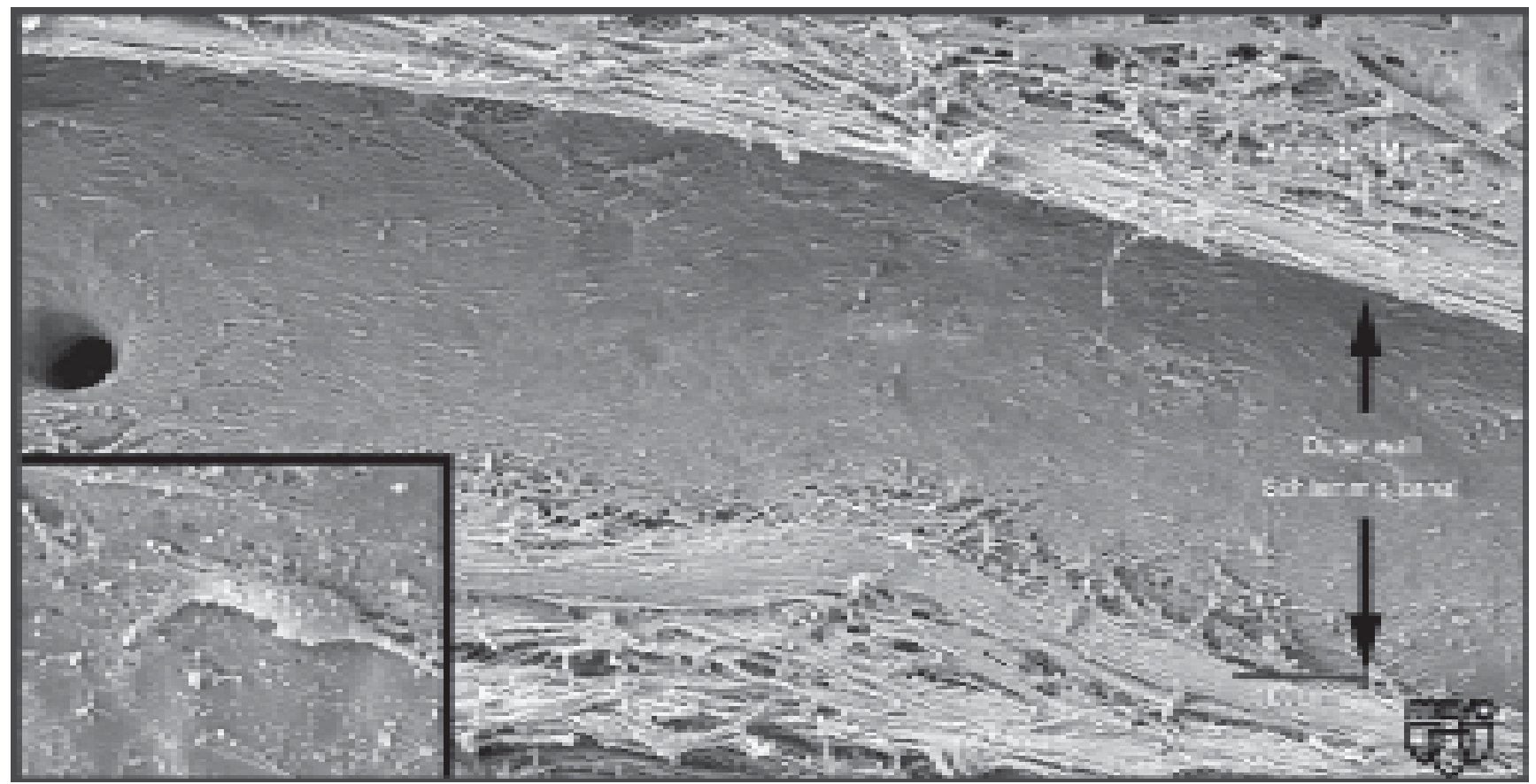

Fig. 4: Trabectome ${ }^{\mathrm{TM}}$ removal of meshwork. Cells visible on outer wall of Schlemm's canal (Courtesy of Doug Johnson)

The Trabectome ${ }^{\mathrm{TM}}$ procedure has also been combined with cataract extraction (phacoemulsification and IOL placement) in approximately 100 cases with reasonable IOP improvement.

Skill transfer has been easy among the approximately 25 surgeons so far involved. This procedure probably best fits into current management schemes for open-angle glaucoma between laser trabeculoplasty and standard filtering procedures. No prospective trials comparing Trabectome ${ }^{\mathrm{TM}}$ to standard filtering surgery or to medicine and laser have yet been performed.

(Disclosure: Minckler is a paid consultant for NeoMedix, manufacturer of the Trabectome ${ }^{\mathrm{TM}}$ ).

\section{REFERENCES}

1. Minckler DS, Baerveldt G, Alfaro MR, Francis BA. Clinical results with the trabectome for treatment of open-angle glaucoma. Ophthalmology 2005;112;962-7.

2. Minckler DS, Baerveldt G, Ramirez M, Mosaed S, Wilson R, Shaarawy T, Dustin L, Francis BA. Clinical results with the trabectome, a novel surgical device for treatment of adult openangle glaucoma. Transactions of the American Ophthalmological Society, 2007;CIV, V:104.

3. Francis BA, See RF, Rao NA, Minckler DS, Baerveldt G. Ab interno trabeculectomy: development of a Novel device (Trabectome) and surgery for open-angle glaucoma. Journal of Glaucoma 2006;15:68-73. 


\title{
Submitting a paper/video to Journal of Current Glaucoma Practice (Glaucoma Review Journal with DVD)
}

\begin{abstract}
INSTRUCTION TO AUTHORS
Journal of current glaucoma practice is a glaucoma review journal with video assisted teaching. It publishes invited review articles on basic and clinical sciences in Glaucoma and videos related to glaucoma diagnostics or surgery. Original research material can also be integrated into a review article. Articles should be submitted only by individuals with experience and expertise in the topic that they are reviewing. Videos can be submitted by any glaucoma specialist and may be incorporated into the journal DVD after peer review.

Before preparing a manuscript, the author should submit a detailed outline of the proposed article to the Editor-in-Chief to assure that the material is appropriate and that no similar article is in preparation. Please allow 2 to 4 weeks for a response.
\end{abstract}

Please do not hesitate to contact the Editorial Office if you have any questions.

\section{THE REVIEW}

Your review can be up to 2500 words in length and should highlight and discuss all interesting developments in the subject, as reflected in the recent literature. In addition to describing recent trends, you can give a sysnopsis of your own opinions of the topics discussed and suggest a preferred practice pattern.

\section{MANUSCRIPT FORMAT}

The review must be double-spaced and a maximum of 2500 words in length (excluding references).

\section{Review structure}

The review must contain the following:

Cover page: Stating the title, authors and their affiliations, and full contact details for the corresponding author (including phone number and e-mail address).

Structured Abstract: This should be no longer than 200 words and contain the following subheadings:

Purpose of review: Describes why this review is timely and relevant.

Recent findings: Describe the main themes in the literature covered by the article.

Summary: Describes the implications of the findings for clinical practice or research.

Key words: 3-5 key words relevant to the paper should be listed.

Introduction: This should be a paragraph of 50-100 words outlining the scope of the review and mentioning any earlier work which will place the review in context.

Text of review: Includes headings and titled paragraphs to subdivide the text. Ensure that at least one sentence divides each heading (i.e. do not have a subheading directly beneath a full heading).

Conclusion: A paragraph of 50-100 words drawing together the implications of the review topic and, if appropriate, giving suggestions for future research.

Acknowledgements: Of professional colleagues and funding bodies only.

Reference section: References should be in numerical sequence (Vancouver style), include the first three authors, or all authors if there are six or fewer.

Figure titles and legends: Must be provided for all figures

Figures and tables: Must be cited in text.

Figures and tables must be

Original whenever possible

Clearly marked as "original" or "previously published" upon submission

Accompanied by full source details when not original

Figures should not be embedded within the text but should be submitted as separate files. Figure legends should be included in the main body of the text following the references.

References cited in figures or tables must be numbered in sequence, according to the position of the first text citation of the figure or table.

\section{Illustrations}

Original ideas for explanatory diagrams are welcomed. Electronic artwork should be submitted in JPEG, TIFF, EPS or PPT formats. Please state clearly whether figures/tables have been published previously.

Color figures: There is a no charge to authors if you wish your figures to be produced in color. 


\title{
DISCLOSURES
}

Authors should include all relevant information regarding Conflict of Interest and Sponsorship Statements.

\section{SUBMITTING YOUR PAPER}

Manuscripts should be submitted by e-mail at currentglaucomapractice@gmail.com

If you have any query or suggestion, please do not hesitate to contact:

Editor-in Chief

Tanuj Dada, MD, Dr Rajendra Prasad Centre for Ophthalmic Sciences, All India Institute of Medical Sciences, New Delhi, India e-mail: tanujdada@hotmail.com

The manuscript file should include the text, references, structured abstract, keywords, figure legends and author address. Figures and tables should be submitted as separate files.

References cited in figures or tables must be numbered in sequence, according to the position of the first text citation of the figure or table. Unpublished data, submitted manuscripts and personal communications must be referenced in the text only as follows:

Personal communication: (Author A, personal communication).

Submitted paper: (Author A, Author B, unpublished data).

You are responsible for the accuracy of the references and for obtaining permission to use personal communications.

\section{REFERENCE FORMAT}

In the text, reference citations should be typed in superscript.

Please list the first three authors for each reference and then et al, unless there are six authors or fewer, in which case all authors should be listed. Provide full reference details (author(s), title, journal, year, volume, pages).

Journal names should be abbreviated as in the Index Medicus.

References should be structured as follows:

Journal:

Author A, Author B, Author C. Title of the paper. Journal Abbreviation 2000; 4:25-27.

With annotation describing importance of reference, if bulleted

Book:

Author A, Author B, Author C. Title of the book section. In: Book name. Edition number. Edited by Editor A, Editor B, Editor C (editors). Location of Publisher: Publisher; 2000. pp. 25-27.

Copyright transfer form with article title and signature of all authors needs to be mailed or faxed during submission of the article.

\section{SUBMISSION OF VIDEOS}

The journal accepts videos demonstrating surgical techniques and newer diagnostic techniques. Videos for the CD/DVD should be edited with an audio commentary or a text overlay and submitted in the following formats: .mpg/.wmv/.dat The CD/DVD with video may be directly sent to the journal editorial office (address given below).

Video should be accompanied by

Cover page: stating the title, authors and their affiliations, and full contact details for the corresponding author (including phone number and email address).

A small summary of the procedure being shown in about 150 words should be included.

Video should be accompanied by a moving legend describing the procedure with or without an audio in English language.

\section{ADDRESS OF THE JOURNAL OFFICE FOR SUBMISSION OF VCD OR DVD VIDEOS:}

\author{
Jaypee Brothers Medical Publishers Pvt. Ltd. 23/23B EMCA House, Ansari Road, Daryaganj, New Delhi, India \\ Phones: +91-11-23272143, +91-11-23272703, +91-11-23282021, +91-11-23245672 \\ Rel: 32558559 Fax: +91-11-23276490, +91-11-23245683 \\ e-mail: jaypee@jaypeebrothers.com
}




\section{SUBSCRIPTION INFORMATION}

\section{Annual subscription:}

Individual: Rs. 1600.00

$\$ 130.00$

£ 75.00

Institutional: Rs. 3000.00

$$
\$ 160.00
$$$$
\text { £ } 100.00
$$

(national)
(international)
(international)
(national)
(international)
(international)

Subscription can be sent to
M/s Jaypee Brothers Medical Publishers (P) Ltd
Journals Department
G-7, 23/23B, EMCA House, Ansari Road
Daryaganj, New Delhi 110 002, India
Phones:+91-11-23272143, +91-11-23272703,
$\quad+91-11-23282021,+91-11-23245672$
Fax: $\quad+91-11-23276490,+91-11-23245683$

Subscription can be sent to M/s Jaypee Brothers Medical Publishers (P) Ltd Journals Department G-7, 23/23B, EMCA House, Ansari Road Daryaganj, New Delhi 110 002, India 91-11-23282021, +91-11-23245672

$+91-11-23276490,+91-11-23245683$

This journal is published thrice in a year, i.e. January, May and September, every year. Dollar rates apply to subscribers in all the countries except the UK and the Republic of Ireland where the pound/sterling price applies. All subscriptions are payable in advance and all the rates include postage. Journals are sent by air to all the countries except Indian subcontinent. Subscriptions are on an annual basis, i.e. from January to December. Payment will be made by sterling cheque, dollar cheque, credit card or directly through our bank account at the following address:
1. Our banker's name:
Canara Bank, Netaji Subhash Marg

2. Telephone No:

Darya Ganj, New Delhi 110002

3. Fax No:

011-23273015, 011-23273849

4. Telex Number:

011-23255606

5. Our Current A/c No:

3166291

\section{8}

6. Amount to be transferred

JAYPEE BROTHERS MEDICAL in the name of:

PUBLISHERS (P) LTD., NEW DELHI

CNRB IN BB DFM

7. Swift code No:

For further queries please do not hesitate to contact MR TARUN DUNEJA at e-mail: t_duneja@rediffmail.com

\section{ADVERTISEMENT RATES}

(For the Print Issues)

Page

Back cover-colour

Inside front cover-colour

Inside back cover-colour

Special position*-colour

Inside full page-colour

\begin{tabular}{ll}
\multicolumn{2}{c}{ Single issue } \\
\hline Rs. 25,000 & $\$ 625.00$ \\
Rs. 20,000 & $\$ 500.00$ \\
Rs. 15,000 & $\$ 375.00$ \\
Rs. 12,500 & $\$ 300.00$ \\
Rs. 10,000 & $\$ 250.00$
\end{tabular}

*First page, page facing editorial board, page facing table of contents.

Cover page advertisements not available for a single issue.

\section{Technical Details}

Paper size

Print size

Digital file format

Printed on art paper using offset printing.

\section{$8.5 \times 11.5$ inches}

$7 \times 10$ inches

EPS on CD (at 300 dpi resolution)

\section{Schedule}

Issues are published in the months of January, May and September.

Advertisement material along with purchase order and payment should reach us at least four weeks prior to the scheduled print date.

\section{Payment Details}

- Payment should favour "Jaypee Brothers Medical Publishers Pvt (Ltd)" and should be payable at New Delhi, India.

- Payment to be done at the time of submitting the advertisement material/booking the advertisement. Please send your advertisement request, payment and advertisement material to the address given above. Editorial board reserves the right to accept or decline the advertisement. 